\title{
Type 3 Autoimmune Lymphoproliferative Syndrome
}

National Cancer Institute

\section{Source}

National Cancer Institute. Iype 3 Autoimmune Lymphoproliferative Syndrome. NCI

Thesaurus. Code C39577.

A rare, primary immunodeficiency. It is caused by a currently undetermined defect in the Fas-induced apoptosis pathway. No mutations in Fas, FASLG or CASP10 are detectable. Disruption of Fas-induced apoptosis impairs lymphocyte homeostasis and immune tolerance. Characteristic laboratory findings include an increase in circulating, doublenegative (CD4-/CD8-) T cells in the setting of immune-mediated anemia, thrombocytopenia and neutropenia. Clinical signs present in childhood include fatigue, pallor, bruising, hepatosplenomegaly and chronic, non-malignant, non-infectious lymphadenopathy. The clinical course is influenced by a strong association with other autoimmune disorders and an increased risk for developing Hodgkin and non-Hodgkin lymphoma. 